\title{
Comparison of Abbott LCx Chlamydia trachomatis Assay With Gen-Probe PACE2 and Culture
}

\author{
David C. Young, ${ }^{1 *}$ Sandra Craft, ${ }^{2}$ Mary-Clare Day, ${ }^{3}$ Barbara Davis, ${ }^{2}$ \\ Elizabeth Hartwell, ${ }^{1}$ and Song Tong ${ }^{1}$ \\ ${ }^{1}$ Department of Pathology, University of Texas Health Science Center at Houston, TX \\ ${ }^{2}$ Dynacare/Hermann Laboratories, Houston, TX \\ ${ }^{3}$ Department of Obstetrics, Gynecology, and Reproductive Sciences, University of Texas Health \\ Science Center at Houston, TX
}

\begin{abstract}
In this study the LCx assay (a nucleic acid amplification assay) for Chlamydia trachomatis in endocervical samples was compared with the Gen-Probe PACE2 assay (a nucleic acid probe assay) for endocervical samples, and with endocervical culture. In addition, the efficacy of the LCx assay was determined for midstream clean-catch urine samples because it is often necessary to obtain such a sample for routine urine culture and it is simpler to collect only a single sample without also collecting a first-void urine for LCx. Endocervical specimens from 205 patients were tested for $C$. trachomatis via LCx and PACE2. Of these patients, 203 were tested by culture. Midstream cleancatch urine samples from 75 of these patients were tested by LCx. The sensitivities and specificities for these assays, after discrepant analysis, were 100 and $98.9 \%$ for LCx of endocervical samples, 52.4 and $100 \%$ for PACE2; and 71.4 and $100 \%$ for culture. The sensitivity/specificity of LCx for midstream clean-catch urines was $66.7 / 98.5 \%$. The apparent prevalence of $C$. trachomatis in our population was $10.2 \%$. These data indicate that among the methods tested, LCx of endocervical samples had the highest sensitivity for $C$. trachomatis in this population. The senstivity of the urine LCx assay using midstream clean-catch collected urines was considerably less than that reported in other studies that used first-void urines but was higher than that of PACE2. Infect. Dis. Obstet. Gynecol. 8:112-115, 2000. @ 2000 Wiley-Liss, Inc.
\end{abstract}

KEY WORDS

diagnostic tests; sensitivity; specificity

\footnotetext{
$c$
} hlamydia trachomatis $(\mathrm{Ct})$ is the most common sexually transmitted bacterial infection in the United States with an incidence of about 4-5\% in the young adult population. ${ }^{1,2}$ It causes asymptomatic infections in as many as $25 \%$ of men $^{3,4}$ and in $75 \%$ of women, ${ }^{5}$ which leads to many undetected cases. It also leads to significant morbidity, giving a relative risk of ectopic pregnancy of 2.4-7.96 and causing epididymitis in approximately $3 \%$ of infected males. ${ }^{7}$ These characteristics, and the fact that this disease is readily treatable make it an excellent candidate for a screening program. There are a variety of available methods with which to screen for Ct including culture, the Gen-Probe PACE2 assay (a nucleic acid probe assay), and more recently, LCx. The LCx assay which is based

Grant sponsor: Dynacare/Hermann Laboratories; Grant sponsor: Abbott Laboratories.

*Correspondence to: David C. Young, University of Texas Health Science Center, 6431 Fannin Street, MSMB 2.137, Houston, Texas 77030. E-mail: young@casper.med.uth.edu 
on the ligase chain reaction ${ }^{8}$ is expected to have high sensitivity since it is a nucleic acid amplification assay, and to have high specificity because it involves the use of four probes. In addition, LCx is suitable for use with male and female urine samples whereas PACE2 and culture are not. In this study the diagnostic efficiency of the LCx assay was compared with that of the PACE2 assay and with culture, and the effects on LCx of substituting midstream clean-catch urine samples for first-void samples was evaluated.

\section{METHODS \\ Study Population}

Specimens were collected for Ct testing at the Hermann Ob/Gyn Clinic of the University of Texas Health Science Center in Houston, Texas. The mean age of the patients was 24.3 years with a range of 15-49 years. Approximately $90 \%$ of these patients were undergoing prenatal evaluation. Most of the remainder were seen for routine checkups.

\section{Specimen Collection}

In all, 216 specimens were obtained; one specimen for each type of assay was collected from each patient. Nine specimens were eliminated from the study because of use of the wrong swab for LCx collection. Two specimens were eliminated due to lack of Gen-Probe and culture results. Specimens for LCx, PACE2, and culture were collected simultaneously from each patient. LCx and PACE2 collection of endocervical samples was according to manufacturer's instructions. Cervical samples for Ct culture were collected by use of Dacron polyester-tipped swabs with polystyrene handles (Hardwood Products, Guilford, ME) and placed in transport medium (Multi-Microbe Media, Micro Test Inc. Lilburn, GA). Midstream clean-catch urine samples that showed evidence of leukocytes by urine dipstick were tested for Ct by LCx. All patients were evaluated for pyuria by testing midstream clean-catch urine samples for leukocyte esterase using urine dipsticks. Urine samples that showed evidence of pyuria were sent for routine urine culture and we obtained aliquots of those samples for the LCx assay.

\section{Diagnostic Tests}

Specimens were tested for Ct by use of the Abbott LCx assay (Abbott Laboratories) the PACE2 assay
(Gen-Probe Inc. San Diego, CA), and by culture. Ct culture ${ }^{9}$ was performed by inoculation of specimens onto McCoy cell monolayers (Intracel Inc., Issaquah, WA) in shell vials with detection by IFA using Micro Trak Culture Confirmation Reagent (Syva Co., Palo Alto, CA) according to the manufacturer's recommendations. Our costs per test, including labor, were $\$ 7$ for LCx, $\$ 11$ for Gen-Probe, and $\$ 13$ for culture.

\section{Discrepant Analysis}

Samples positive for Ct by LCx and culture were considered true positives. Samples positive by LCx and negative by culture were retested by LCx at Abbott Laboratories. Samples that were negative by LCx at Abbott were considered false positives. Samples that were positive by LCx at Abbott were retested at Abbott by a ligase chain reaction assay for the major outer membrane protein gene of $\mathrm{Ct}$ (MOMP LCR) and were considered positive or negative based on this result.

Samples found positive by culture were considered true positives. Samples found negative by culture that were true positives for LCx were considered culture false negatives. Negative PACE2 samples that were considered true positives by LCx or culture were considered PACE2 false negatives. No samples were PACE2 positive, LCx negative.

\section{RESULTS}

A total of 205 patients were evaluated for the presence of Ct via endocervical swab by use of LCx and PACE2. Twenty-three cervical samples were positive by LCx. Discrepant analysis showed two of these to be false positives (Table 1). Of the 21 samples that were found to be true positives by LCx, 11 were also positive by PACE2. No samples that were negative by $\mathrm{LCx}$ were positive by PACE2. Of 75 urine samples that were evaluated for Ct by LCx, 7 were positive. Discrepant analysis showed 1 positive to be a false positive and 3 of the 68 negatives were false negatives. One patient was positive by LCx for $\mathrm{Ct}$ in the cervical sample but negative for Ct by LCx of urine.

Of 203 endocervical samples that were tested for Ct by culture, 15 were positive. Discrepant analysis showed 6 of the 188 negatives to be false negatives. No endocervical samples that were negative by LCx were positive by any other method. These 
TABLE I. Performance of LCx, PACE2, and culture for detection of Chlamydia trachomatis after discrepant analysis

\begin{tabular}{|c|c|c|c|c|c|c|c|c|c|}
\hline Test & Source & $\begin{array}{c}\text { True } \\
\text { positive }\end{array}$ & $\begin{array}{c}\text { False } \\
\text { positive }\end{array}$ & $\begin{array}{c}\text { True } \\
\text { negative }\end{array}$ & $\begin{array}{c}\text { False } \\
\text { negative }\end{array}$ & $\begin{array}{c}\text { Sensitivity } \\
(\%)\end{array}$ & $\begin{array}{c}\text { Specificity } \\
(\%)\end{array}$ & $\begin{array}{c}\text { Positive } \\
\text { predictive } \\
\text { value } \\
(\%)\end{array}$ & $\begin{array}{c}\text { Negative } \\
\text { predictive } \\
\text { value } \\
(\%) \\
\end{array}$ \\
\hline LCx & Cervix & 21 & 2 & 182 & 0 & 100 & 98.9 & 91 & 100 \\
\hline LCx & Urine & 6 & I & 65 & 3 & 66.7 & 98.5 & 85.7 & 95.6 \\
\hline PACE2 & Cervix & 11 & 0 & 194 & 9 & 52.4 & 100 & 100 & 95.4 \\
\hline Culture & Cervix & 15 & 0 & 182 & 6 & 71.4 & 100 & 100 & 96.8 \\
\hline
\end{tabular}

results are summarized in Table 1 where sensitivities, specificities, and positive and negative predictive values are given. The overall incidence of $\mathrm{Ct}$ detected in this population after discrepant analysis was $10.2 \%$.

Two endocervical samples that were positive by LCx were negative by discrepant analysis. One of these samples was also positive by urine LCx. It should be noted, however, that samples were stored at $-20^{\circ} \mathrm{C}$ for approximately 6 months before discrepant analysis was done, whereas the LCx package insert indicates that samples should be stored for no more than 2 months at $-20^{\circ} \mathrm{C}$ before analysis by the standard LCx assay. Samples were, however, heated to $97^{\circ} \mathrm{C}$ for $15 \mathrm{~min}$ prior to storage, per package insert, and were stored in a frost-free freezer. Heating should have destroyed most DNAase. Because samples were not stored in a frost-free freezer they did not undergo the freezethaw cycles that might cause sample degradation. Discrepant analysis involved use of MOMP LCR that detects a single-copy chromosomal gene, whereas LCx detects a plasmid that is present in 7-10 copies per chromosome. ${ }^{10}$ Therefore it is likely that the LCx assay had greater sensitivity than the MOMP LCR that was used for confirmation.

\section{DISCUSSION}

Based on a review of the literature, this is the only direct comparison of LCx with the Gen-Probe PACE2 assay in which conflicting results were resolved by discrepant analysis. We found LCx of endocervical samples to be considerably more sensitive for detection of $\mathrm{Ct}$ than the other methods examined. LCx of cervical samples was $28.6 \%$ more sensitive than culture and PACE2 had a sensitivity of only $52.4 \%$. It should be borne in mind that the LCx assay is based on the amplification of nucleic acid and has been shown to be capable of detecting $\mathrm{Ct}$ in urine as long as 12 days after appropriate therapy, leading to the recommendation that this test not be used for test of cure within 3 weeks of treatment. ${ }^{11}$

Others have shown that LCx of first-void urines (the first 5-20 $\mathrm{ml}$ at the beginning of micturation) had a sensitivity of 93.8 to $100 \% .^{8,12}$ Both first-void urine collections for $\mathrm{LCx}^{8,12}$ and the midstream clean-catch specimens that we studied were collected at variable times of the day and were not necessarily the first void of the day. The high sensitivity of LCx with first-void urines suggested the possibility that midstream urines collected for routine urine culture might serve as adequate samples for LCx which would simplify sample collection. Interestingly, LCx of midstream clean-catch urines had a sensitivity of $66.7 \%$ which was considerably less than the sensitivities obtained by others using first-void urines but was greater than that of PACE2. Thus the sensitivity of LCx for first-void urines appeared to be considerably greater than that for midstream clean-catch urines though the number of urine samples we examined was relatively small. It appears likely that this difference was due to loss of the urethral organisms that are purposefully discarded with the first-void urine when obtaining a midstream clean-catch urine sample.

Because the urine samples that were tested by LCx were selected based on the presence of leukocytes they represent a separate subpopulation. Chow et al. found leukocyte urine esterase to be $53.1 \%$ sensitive and $67.3 \%$ specific for $\mathrm{Ct}$ compared to $\mathrm{LCx} .{ }^{13}$ Thus the leukocyte esterase test was inadequate for use in screening for $\mathrm{Ct}$, but because of selection based on this test the collection of urine samples we received would be expected to be enriched for $\mathrm{Ct}$ positive samples. This appears 
to have been the case since $13.8 \%$ of patients with pyuria were positive for $\mathrm{Ct}$ whereas $9.3 \%$ of patients without pyuria were positive for Ct. Therefore it was of interest to compare the urine LCx results with the results obtained by the other detection methods in this subpopulation. The sensitivity/specificity in this subpopulation was 100/ 98.5\% for LCx of endocervical samples, 50/100\% for culture, and 33/100\% for PACE2. Because of the smaller number of samples in the urine subpopulation, the differences between the urine subpopulation and the total population are probably not significant.

Our results indicate that LCx of endocervical samples had a much higher sensitivity than did the PACE2 assay or culture (which were the methods routinely used in our laboratory). Our limited data does not support routine substitution of midstream clean-catch urine samples for first-void urines in the LCx assay.

\section{REFERENCES}

1. Brodine SK, Shafer MA, Shaffer RA, et al. Asymptomatic sexually transmitted disease prevalence in four military populations: application of DNA amplification assays for Chlamydia and gonorrhea screening. J Infect Dis 1998;178:1202-1204.

2. Control CfD. Recommendations for the prevention and management of Chlamydia trachomatis infections. MMWR Morb Mortal Wkly Rep 1993;42:1-39.

3. Stamm WE, Koutsky LA, Benedetti JK, Jordan JL, Brunham RC, Holmes KK. Chlamydia trachomatis urethral infections in men. Prevalence, risk factors, and clinical manifestations. Ann Intern Med 1984;100:47-51.
4. Stamm WE, Cole B. Asymptomatic Chlamydia trachomatis infection in men. Sex Transm Dis 1986;13:163-165.

5. Schachter J, Stoner E, Moncada J. Screening for chlamydial infections in women attending family planning clinics. West J Med 1983;138:375-379.

6. Cates W, Wasserheit JN. Genital chlamydial infections: epidemiology and reproductive sequelae. Am J Obstet Gynecol 1991;164:1771-1781.

7. Kaufman RD. Nonspecific urethritis. N Engl J Med 1974;289:1175-1177.

8. Lee HH, Chernesky MA, Schachter J, et al. Diagnostic of Chlamydia trachomatis genitourinary infection in women by ligase chain reaction assay of urine. Lancet 1995;345:213-216.

9. Thomas BJ, Evans RT, Hutchinson GR, TaylorRobinson D. Early detection of Chlamydia isolations combining the use of cycloheximide treated McCoy cells and immunofluorescence staining. J Clin Microbiol 1977;6:285-292.

10. Palmer L, Falkow S. A common plasmid of Chlamydia trachomatis. Plasmid 1986;16:52-63.

11. Gaydos CA, Crotchfelt KA, Howell MR, Kalian S, Hauptman P, Quinnn TC. Molecular amplification assays to detect chlamydial infections in urine specimens from high school female students and to monitor the persistence of chlamydial DNA after therapy. J Infect Dis 1998;177:417-424.

12. Gaydos A, Yun F, Lee H, et al. Urine as a diagnostic specimen for the detection of Chlamydia trachomatis in Malaysia by ligase chain reaction. Sex Transm Dis 1995; 23:402-406.

13. Chow J, Moncada J, Brooks D, Bolan G, Shaw H, Schacter J. Is urine leukocyte esterase test a useful screening method to predict Chlamydia trachomatis infection in women? J Clin Microbiol 199634:534-536. 


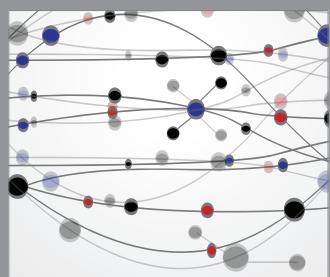

The Scientific World Journal
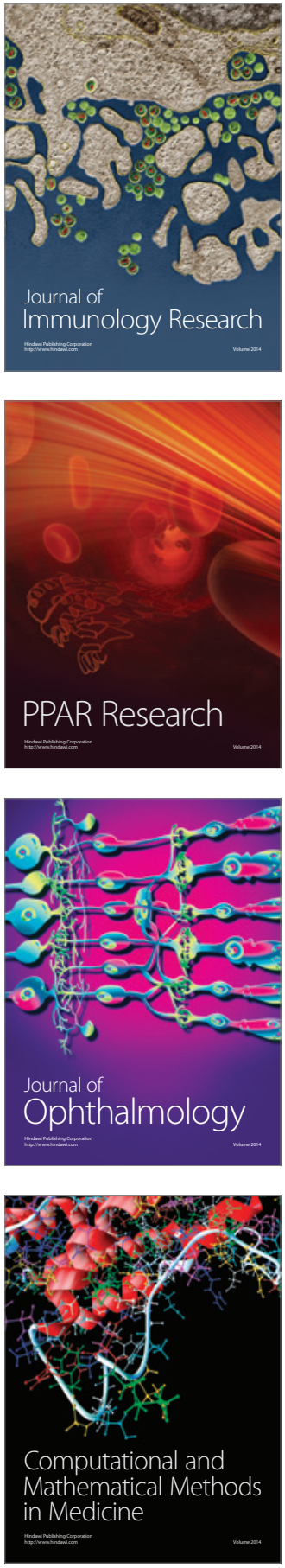

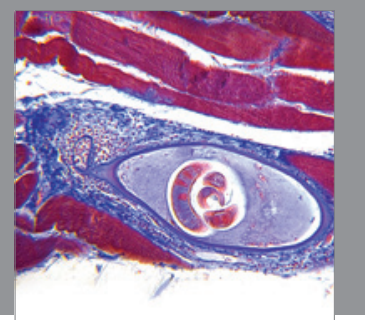

Gastroenterology

Research and Practice
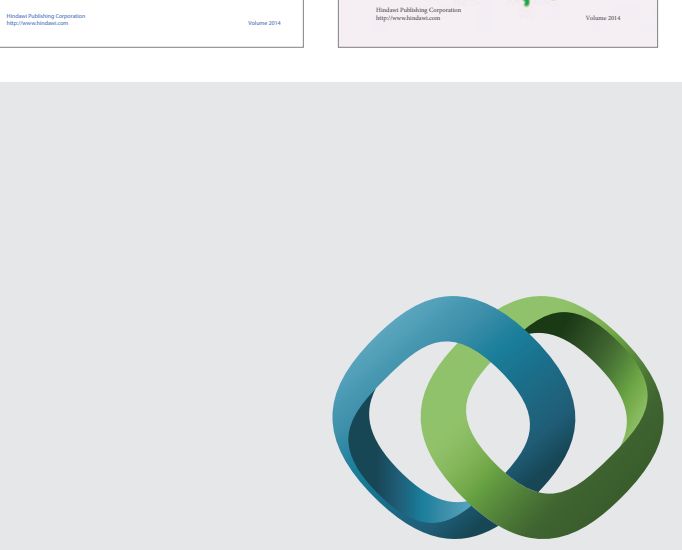

\section{Hindawi}

Submit your manuscripts at

http://www.hindawi.com
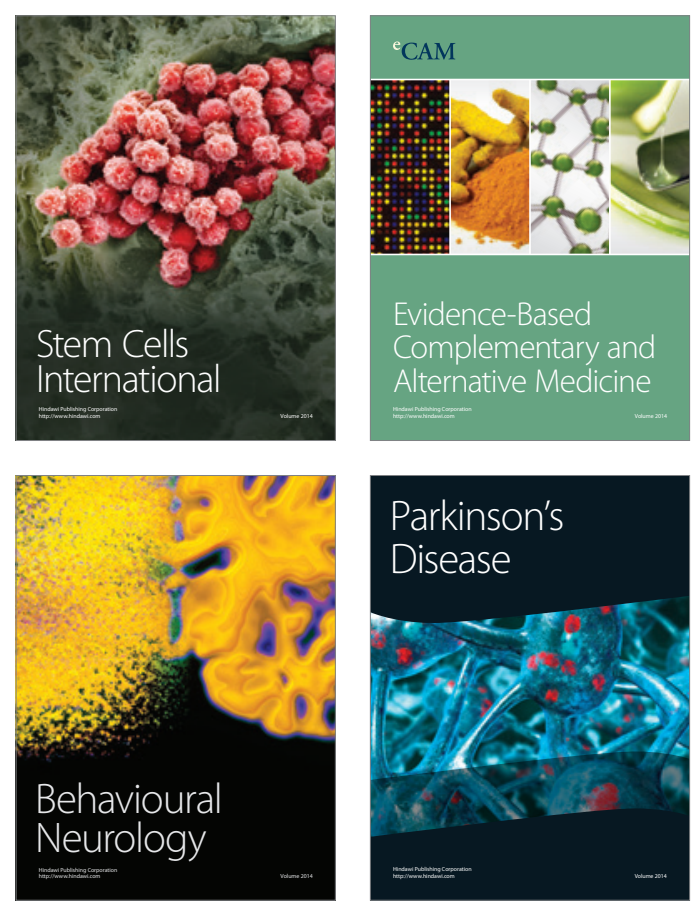

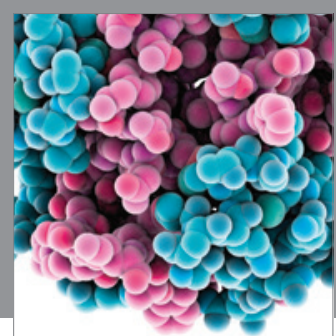

Journal of
Diabetes Research

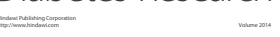

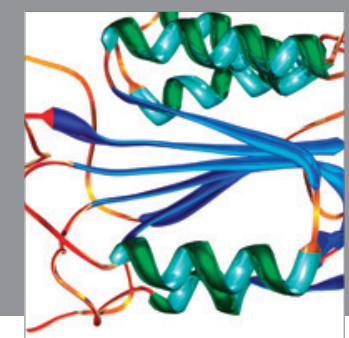

Disease Markers
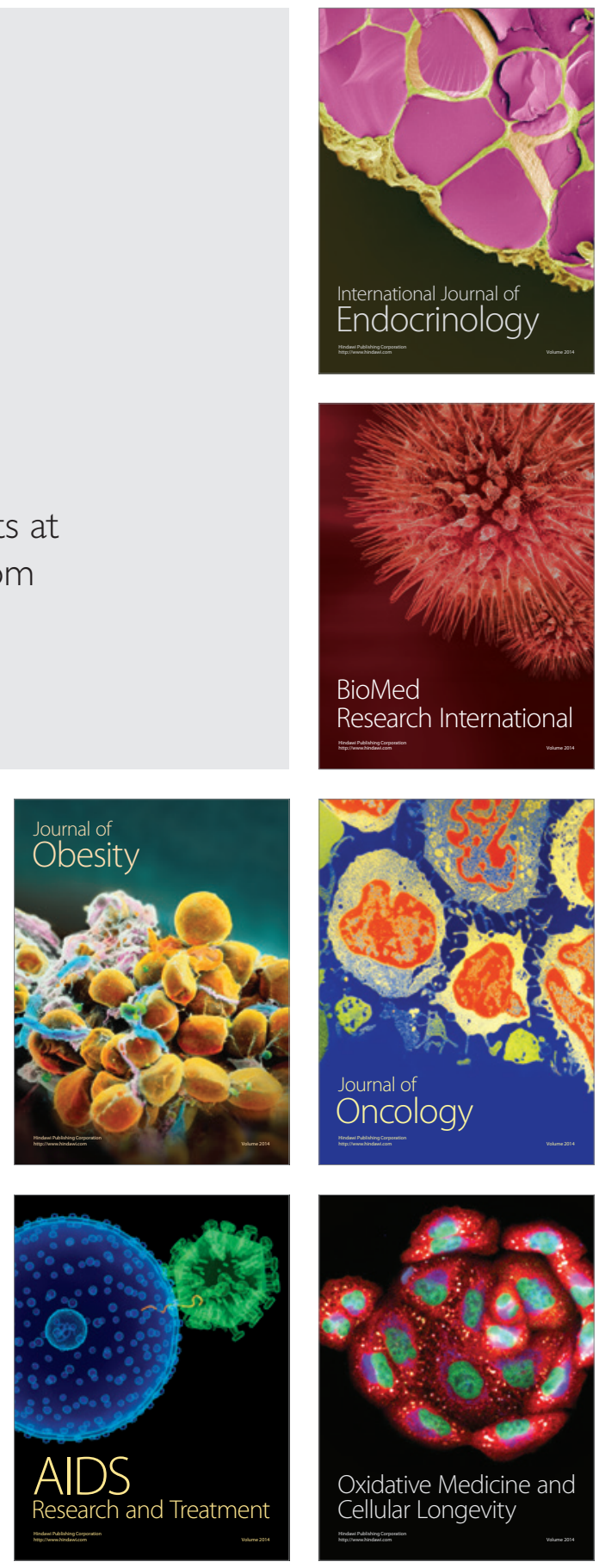